\title{
2,5-取代-1,3,4-噁二唑衍生物的合成与杀菌活性
}

\author{
刘建超＼cjkstart王卫东＼cjkstart贺红武*
}

(华中师范大学化学学院 武汉 430079)

\begin{abstract}
摘要 利用生物活性亚结构拼接原理, 将吡啶环、噻唑环引入到 1,3,4-噁二唑母体结构中, 设计并合成了一系列新型含 吡啶(噻唑)的 1,3,4-啞二唑衍生物. 通过 IR, ${ }^{1} \mathrm{H}$ NMR, EI-MS 及元素分析等方法对所合成的化合物进行了结构表征. 代 表化合物 2-(6-氯吡啶-3-甲硫基)-5-(吡啶-4-基)-1,3,4-啞二唑(I)经单晶 X 衍射证实了结构. 初步测定了所合成化合物的 杀菌活性, 并比较了在 1,3,4-腎二唑母体结构中引入噻唑杂环和引入吡啶杂环后其杀菌活性的差异. 结果表明: 目标化 合物对测试的 5 种菌均具有一定的杀菌活性，对水稻纹枯病的抑制效果普遍优于对其它菌种的抑制效果; 在 1,3,4-惡二 唑母体结构中引入噻唑杂环比引入吡啶杂环对其杀菌活性更有利.
\end{abstract}

关键词 1,3,4-噁二唑; 吡啶环; 噻唑环; 杀菌活性

\section{Synthesis and Fungicidal Activity of 2,5-Disubstituted- 1,3,4-oxadiazole Derivatives}

\author{
Liu, Jianchao Wang, Weidong He, Hongwu* \\ (College of Chemistry, Central China Normal University, Wuhan 430079)
}

\begin{abstract}
A series of new 2,5-disubstituted-1,3,4-oxadiazole derivatives have been designed and synthesized by adding pyridine ring and thiazole ring to the structure of 1,3,4-oxadiazole. The structures of target compounds have been confirmed by ${ }^{1} \mathrm{H}$ NMR, EI-MS, IR spectroscopy and elemental analyses. The structure of 2-[(6-pyridin-3-yl)methylthio]-5-(pyridin-4-yl)1,3,4-oxadazole has been determined by single crystal X-ray diffraction. The results of preliminary bioassay indicated that some compounds possess potential fungicidal activities against Fusarium oxyporium, Gibberella zeae, Rhizoctonia solani, Dothiorella gregaria and Colletotrichum gossypii at a dosage of $5.0 \times 10^{-5} \mathrm{~g} / \mathrm{mL}$. The fungicidal activities of 1,3,4-oxadiazole derivatives adding thiazole ring were better than those of those adding pyridine ring.

Keywords 1,3,4-oxadiazole; pyridine ring; thiazole ring; fungicidal activity
\end{abstract}

自 Gibson ${ }^{[1]}$ 发表论文详尽地论述了 1,3,4-惡二唑衍 生物的环合机理以来，噁二唑衍生物的发展十分迅速， 在不断合成具有各种新颖结构的噁二唑衍生物的过程 中, 出现了很多有效的合成方法, 同时人们还发现这类 化合物具有广泛而优良的生物活性, 如杀虫、除草、杀 菌、抗癌、抗炎等 ${ }^{[2 \sim 5]}$. 在噁二唑母体结构中引入其它 杂环, 产生了众多有杀菌活性的 1,3,4-惡二唑衍生物. 2001 年 Liu 等 ${ }^{[6]}$ 报道了将 1,2,4-[1,5- $a$ 嘧啶环引入 1,3,4啞二唑结构中, 所合成化合物对立枯丝核菌有较高的活 性. 2004 年 Wang 等 ${ }^{[7]}$ 报道了含甲硫基吡唑的 1,3,4-噁二 唑化合物对天冬茎点霉菌(phoma aspavagi sace)和苹果
轮纹病菌 (Physalospora Piricola) 的抑制率都在 $85 \%$ 以 上. 亦有学者将吡啶环、取代苯环、噻二唑、哒嗪、吡 喃酮等引入 1,3,4-噁二唑结构中获得了具有良好生物活 性的 1,3,4-噁二唑衍生物 ${ }^{[8 \sim 12]}$, 但尚未有将噻唑环引入 1,3,4-惡二唑母体结构的报道. 为了进一步寻找新型的 生物活性杂环化合物, 我们利用生物活性亚结构拼接原 理, 将吡啶环、噻唑环引入到 1,3,4-噁二唑母体结构中, 设计并合成了一系列新型含吡啶(噻唑)的 1,3,4-噁二唑 衍生物. 通过 IR, ${ }^{1} \mathrm{H}$ NMR, EI-MS 及元素分析等方法对 所合成的化合物进行了结构表征. 代表化合物 2-(6-氯 吡啶-3-甲硫基)-5-(吡啶-4-基)-1,3,4-噁二唑(I)经单晶 $\mathrm{X}$

\footnotetext{
*E-mail: he1208@mail.ccnu.edu.cn; liujc@mail.ccnu.edu.cn

Received March 6, 2014; revised April 3, 2014; published online April 15, 2014

Project supported by the National Program on Key Basic Research Project (973 Program, No. 2010CB126100), the National Natural Science Foundation of China (Nos. 21172090, 31000867) and the Program for Changjiang Scholars and Innovative Research Team in University (No. IRT0953).

国家重点基础研究发展规划(973 计划, No. 2010CB126100)、国家自然科学基金(Nos. 21172090, 31000867)和教育部创新团队项目部分(No. IRT0953) 资助项目.
} 
衍射证实了结构. 初步测定了所合成化合物的杀菌活 性, 并比较了在 1,3,4-噁二唑母体结构中引入噻唑杂环 和引入吡啶杂环后其衍生物杀菌活性的差异. 目标化合 物的合成路线见 Scheme 1 .

\section{1 结果与讨论}

\section{1 结构表征}

目标化合物的红外光谱中，所有特征基团均有明显 的吸收, 苯环上尖而弱的 $\mathrm{C}-\mathrm{H}$ 伸缩振动吸收带在 3060 $\mathrm{cm}^{-1}$ 附近, $\mathrm{CH}_{2}$ 的 $\mathrm{C}-\mathrm{H}$ 伸缩振动处于 $2935 \mathrm{~cm}^{-1}$ 附近. 芳环的骨架振动在 $1460,1500,1580,1600 \mathrm{~cm}^{-1}$ 处出现 多条谱带形成特征吸收峰组, 在 $1460 \mathrm{~cm}^{-1}$ 附近的谱 带强度较强, 其它谱带强度一般较弱. 噁二唑环上 $\mathrm{C}-$ $\mathrm{O}-\mathrm{C}$ 伸缩振动一般位于 1160 和 $1100 \mathrm{~cm}^{-1}$ 附近, 强度 中等. 分析目标化合物的 ${ }^{1} \mathrm{H}$ NMR 数据, 化合物的各个 峰均得到了验证. $\delta 4.44$ 左右处的单峰是 $\mathrm{SCH}_{2}$ 的吸收
峰, $\delta 5.20$ 左右处的单峰是 $\mathrm{OCH}_{2}$ 的吸收峰, $\delta 6.71 \sim 8.82$ 之间的多重峰是芳环上的氢的吸收信号. 部分目标化合 物采用 EI-MS 检测. 从它们的质谱数据可以发现目标分 子均有正常的分子离子峰, 主要碎片离子峰都与预期目 标分子结构吻合. 在元素分析数据中理论值与计算值也 符合得很好, 其相对误差基本在 $0.5 \%$ 范围内. 晶体结构 分析表明，化合物 $\mathbf{I}$ 晶体为三斜晶系，空间群 $P-1$ (图 1).

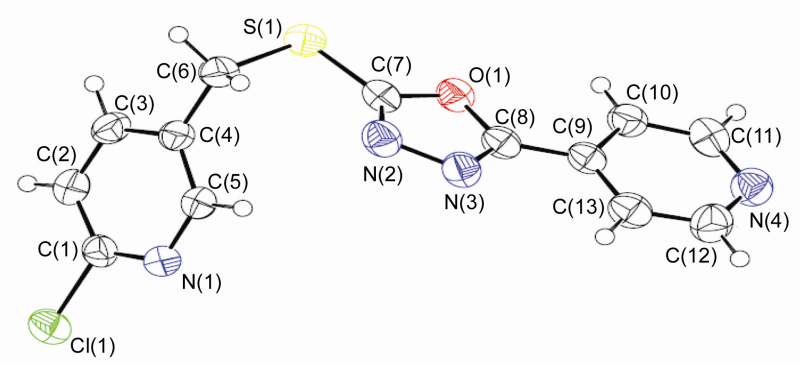

图 1 化合物 $\mathbf{I}$ 的分子结构图

Figure 1 Molecular structure of compound I<smiles>[R]c1nnc(SCc2ccc(Cl)nc2)o1</smiles><smiles>[R]c1nnc(S)o1</smiles><smiles>ONOO</smiles><smiles>[R]c1nnc(SCc2cnc(Cl)s2)o1</smiles>

$A_{1}: R=\mathrm{C}_{5} \mathrm{H}_{4} \mathrm{~N}_{4}$ $A_{2}: \mathrm{R}=4-\mathrm{CH}_{3} \mathrm{OC}_{6} \mathrm{H}_{4}$

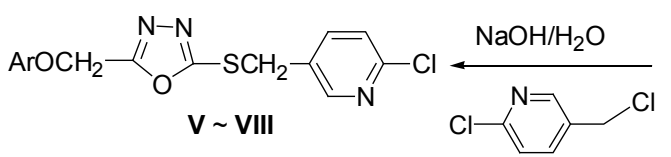

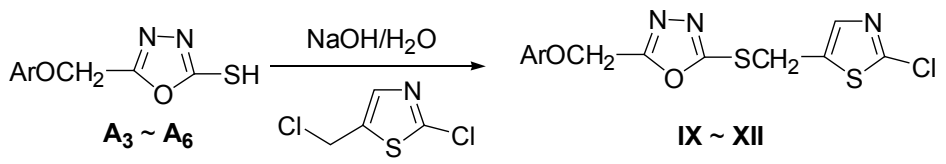
$\mathrm{A}_{3}: \mathrm{Ar}=2,4-\mathrm{Cl}_{2} \mathrm{C}_{6} \mathrm{H}_{3}$
$\mathbf{A}_{4}: \mathrm{Ar}=2-\mathrm{ClC}_{6} \mathrm{H}_{4}$
$\mathrm{A}_{5}: \mathrm{Ar}=4-\mathrm{ClC}_{6} \mathrm{H}_{4}$
$A_{6}: \mathrm{Ar}=3-\mathrm{ClC}_{6} \mathrm{H}_{4}$

\section{Scheme 1}

表 1 目标化合物的抑菌活性数据 $\left(5.0 \times 10^{-5} \mathrm{~g} / \mathrm{mL}\right)^{a}$

Table 1 Fungicidal activity of target compounds $\left(5.0 \times 10^{-5} \mathrm{~g} / \mathrm{mL}\right)$

\begin{tabular}{lccccc}
\hline \multirow{2}{*}{ Compound } & \multicolumn{5}{c}{ Inhibition rate/\% } \\
\cline { 2 - 6 } & Fusarium oxysporium & Rhizoctonia solani & Gibberella zeae & Dothiorella gregaria & Colletotrichum gossypii \\
\hline I & 69.57 & 79.47 & 64.00 & 50.00 & 67.83 \\
II & 17.39 & 28.42 & 14.00 & 18.18 & 17.39 \\
III & 97.83 & 94.74 & 92.05 & 77.72 & 82.61 \\
IV & 46.09 & 71.58 & 38.12 & 39.90 & 30.43 \\
V & 30.43 & 78.95 & 56.00 & 39.90 & 65.22 \\
VI & 26.09 & 52.63 & 24.24 & 22.73 & 43.48 \\
VII & 21.74 & 16.84 & 16.00 & 27.27 & 30.43 \\
VIII & 21.74 & 36.84 & 20.00 & 22.73 & 43.48 \\
IX & 56.09 & 79.68 & 68.00 & 31.82 & 76.52 \\
X & 56.52 & 82.11 & 68.00 & 72.73 & 65.22 \\
XI & 52.17 & 76.84 & 64.00 & 59.09 & 69.57 \\
XII & 52.17 & 66.32 & 68.00 & 63.64 & 69.59 \\
\hline
\end{tabular}

此表数据由华中师范大学化学学院生测室提供. 


\section{2 化合物的杀菌活性}

将目标化合物配制成浓度为 $5.0 \times 10^{-5} \mathrm{~g} / \mathrm{mL}$ 药液, 采用离体平血法对棉花枯萎菌(Fusarium oxyporium)、水 稻纹枯菌(Rhizoctonia solani)、小麦赤霸菌(Gibberella zeae)、苹果轮纹菌(Dothiorella gregaria) 和棉花炭疽菌 (Colletotrichum gossypii)等 5 种菌进行了初步杀菌活性 测试, 具体测试方法见文献[13,14], 测试结果见表 1 .

上述初步生测结果表明: 目标化合物对以上 5 种菌 均具有一定的杀菌活性, 其中对水稻纹枯菌的抑制率普 遍优于对其他几种菌种, 大多数抑制率都在 $70 \%$ 以上. 特别是化合物 III, 对所测试菌种均有显著的抑制活性, 抑制率在 $77.72 \% \sim 97.83 \%$ 之间，对棉花枯萎、水稻纹 枯、小麦赤霉菌的抑制率都达到了 $92.00 \%$ 以上. 从表 1 中可以看出, 化合物 III 的活性优于化合物 I, 化合物 IV 的活性也要比化合物 II 好; 化合物 IX $\sim$ XII 的活性抑制 率分别略高于化合物 IV VIII, 说明在 1,3,4-惡二唑母 体结构中引入噻唑杂环比引入吡啶杂环, 其衍生物的杀 菌活性有利.

\section{2 结论}

利用生物活性亚结构拼接原理, 将吡啶环、噻唑环 引入到 1,3,4-噁二唑母体结构中, 设计并合成了一系列 新型含吡啶(噻唑)的 1,3,4-噁二唑衍生物. 通过 IR, ${ }^{1} \mathrm{H}$ NMR, EI-MS 及元素分析等方法对所合成的化合物进行 了结构表征. 采用离体平皿法对棉花枯萎菌 (Fusarium oxyporium)、水稻纹枯菌(Rhizoctonia solani)、小麦赤雱 菌(Gibberella zeae)、苹果轮纹菌(Dothiorella gregaria) 和棉花炭疽菌(Colletotrichum gossypii) 等 5 种菌进行了 初步杀菌活性测试. 结果表明: 目标化合物对测试的 5 种菌均具有一定的杀菌活性, 尤其对水稻纹枯菌, 其抑 制率优于其他几种菌种, 基本上在 $70 \%$ 以上; 在 1,3,4噁二唑母体结构中引入噻唑杂环比引入吡啶杂环, 其杀 菌活性更有利.

\section{3 实验部分}

\section{1 仪器与试剂}

巩义市英峪予华仪器厂 SHZ-D (III)型循环水式真 空原; 北京泰克仪器有限公司 X-4 型数字显微熔点测定 仪(温度未校正); 德国 BÜchi Rotavapor R-200 旋转蒸发 仪; 德国 Heidilph MR 3001 磁力搅拌器; 美国 Nicolet AVATAR-360 型红外光谱仪( $\mathrm{KBr}$ 压片); 美国 Varian Mercury 400 型 $400 \mathrm{MHz}$ 超导核磁共振仪 (以 $\mathrm{CDCl}_{3}$ 为溶 剂, TMS 为内标); Finnigan Trace MS 质谱仪; Vario EL III 元素分析仪; Brucker APEX CCD Dector 型 X-Ray 单 晶衍射仪. 试剂均为市售分析纯或化学纯, 2-氯-5-氯甲
基吡啶由武汉申试化工仪器网络有限公司提供, 含量在 97\%以上，使用前未做进一步提纯.

\section{$3.2 \mathrm{~A} 1 \sim \mathrm{A} 6$ 的制备}

5-取代-1,3,4-噁二唑-2-硫醇 $\mathbf{A 1} \sim \mathbf{A 6}$ 的合成参见文 献 $[15,16]$.

\section{3 目标化合物的制备}

以化合物 I 的合成为例. 于 $50 \mathrm{~mL}$ 圆底烧瓶中加入 5-取代-1,3,4-噁二唑-2-硫醇(A1) $(3.0 \mathrm{mmol}), 0.13 \mathrm{~g}$ (3.3 $\mathrm{mmol}) \mathrm{NaOH}$ 的 $15 \mathrm{~mL}$ 水溶液, 搅拌溶解后, 室温下缓

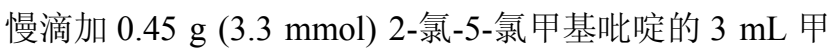
醇溶液, 室温摚拌, TLC 监控至原料点消失, 停止反应, 抽滤，乙醇和水的混合溶剂重结晶或柱层析(丙酮石油 醚梯度洗脱)得纯品. 参照上述方法可以合成目标化合 物 II $\sim$ XII

2-(6-氯吡啶-3-甲硫基)-5-(吡啶-4-基)-1,3,4-嘒二唑 (I)：白色固体，产率 54\%. m.p. $130.9 \sim 132.7{ }^{\circ} \mathrm{C} ;{ }^{1} \mathrm{H}$ NMR $\left(400 \mathrm{MHz}, \mathrm{CDCl}_{3}\right) \delta: 4.51\left(\mathrm{~s}, 2 \mathrm{H}, \mathrm{CH}_{2}\right), 7.31 \sim 8.82$ (m, 7H, Pyridyl-H); ${ }^{13} \mathrm{C}$ NMR (100 MHz, $\left.\mathrm{CDCl}_{3}\right) \delta: 32.7$, $119.9,124.2,130.3,130.7,139.4,142.8,150.0,151.1$, 164.2, 164.4; IR (KBr) v: $3048(\mathrm{Ar}-\mathrm{H}), 2940,1607$, 1587, 1459, 1190, $1105(\mathrm{C}-\mathrm{O}-\mathrm{C}), 700 \mathrm{~cm}^{-1}$. Anal. calcd for $\mathrm{C}_{13} \mathrm{H}_{9} \mathrm{ClN}_{4} \mathrm{OS}$ : C 51.23, H 2.98, N 18.38; found C 51.28, H 2.69, N 18.63.

2-(6-氯吡啶-3-甲硫基)-5-(4-甲氧基苯基)-1,3,4-噁 二唑(II): 白色固体, 产率 71\%. m.p. 125.1 126.1 ${ }^{\circ} \mathrm{C}$; ${ }^{1} \mathrm{H}$ NMR (400 MHz, $\left.\mathrm{CDCl}_{3}\right) \delta: 3.88\left(\mathrm{~s}, 3 \mathrm{H}, \mathrm{CH}_{3}\right), 4.46$ (s, $\left.2 \mathrm{H}, \mathrm{CH}_{2}\right), 6.98 \sim 8.49(\mathrm{~m}, 7 \mathrm{H}, \mathrm{Ar}-\mathrm{H})$; EI-MS $(70 \mathrm{eV}) \mathrm{m} / \mathrm{z}$ (\%): $333\left(\mathrm{M}^{+}, 46\right), 208$ (6), 175 (6), 135 (100), 126 (44), 107 (9), 77 (19),76 (10); IR (KBr) v: $3051(\mathrm{Ar}-\mathrm{H}), 2938$, 1611, 1587, 1501, 1466, 1256, 1167, $1105(\mathrm{C}-\mathrm{O}-\mathrm{C})$ $\mathrm{cm}^{-1}$. Anal. calcd for $\mathrm{C}_{15} \mathrm{H}_{12} \mathrm{ClN}_{3} \mathrm{O}_{2} \mathrm{~S}: \mathrm{C} 53.97, \mathrm{H} 3.62, \mathrm{~N}$ 12.59; found C 54.03, H3.30, N 12.75.

2-(2-氯噻唑-5-甲硫基)-5-(吡啶-4-基)-1,3,4-聟二唑 (III): 白色固体，产率 56\%. m.p. $103.9 \sim 105.5{ }^{\circ} \mathrm{C} ;{ }^{1} \mathrm{H}$ NMR (400 MHz, $\left.\mathrm{CDCl}_{3}\right) \delta: 4.69\left(\mathrm{~s}, 2 \mathrm{H}, \mathrm{CH}_{2}\right), 7.60 \sim 8.83$ (m, 5H, Ar-H); IR (KBr) v: $3084(\mathrm{Ar}-\mathrm{H}), 2929,1566$, 1470, 1189, $1046(\mathrm{C}-\mathrm{O}-\mathrm{C}) \mathrm{cm}^{-1}$. Anal. calcd for $\mathrm{C}_{11} \mathrm{H}_{7} \mathrm{ClN}_{4} \mathrm{OS}_{2}$ : C 42.51, H 2.27, N 18.03; found C 42.62, $\mathrm{H} 2.07, \mathrm{~N} 18.32$.

2-(2-氯噻唑-5-甲硫基)-5-(4-甲氧基苯基)-1,3,4-噁 二坐(IV): 白色固体, 产率 $74 \%$. m.p. $119.1 \sim 120.3{ }^{\circ} \mathrm{C}$; ${ }^{1} \mathrm{H}$ NMR $\left(400 \mathrm{MHz}, \mathrm{CDCl}_{3}\right) \delta: 3.88\left(\mathrm{~s}, 3 \mathrm{H}, \mathrm{CH}_{3}\right), 4.64$ (s, $2 \mathrm{H}, \mathrm{CH}_{2}$ ), $6.99 \sim 7.95(\mathrm{~m}, 5 \mathrm{H}, \mathrm{Ar}-\mathrm{H})$; IR (KBr) v: 3101, 2994, 2942, 2839, 1611, 1503, 1467, 1173, 1049 (C-OC) $\mathrm{cm}^{-1}$. Anal. calcd for $\mathrm{C}_{13} \mathrm{H}_{10} \mathrm{ClN}_{3} \mathrm{O}_{2} \mathrm{~S}_{2}$ : C 45.95, H 2.97, 
$\mathrm{N}$ 12.37; found C 46.11, H 3.01, N 12.62.

2-(6-氯吡啶-3-甲硫基)-5-(2,4-二氯苯氧亚甲 基)-1,3,4-噁二唑(V): 白色固体，产率 61\%. m.p. 82.1 $84.1{ }^{\circ} \mathrm{C}$; ${ }^{1} \mathrm{H}$ NMR (400 MHz, $\left.\mathrm{CDCl}_{3}\right) \delta$ : 4.44 (s, 2H, $\left.\mathrm{SCH}_{2}\right), 5.26\left(\mathrm{~s}, 2 \mathrm{H}, \mathrm{OCH}_{2}\right), 7.01 \sim 8.48(\mathrm{~m}, 6 \mathrm{H}, \mathrm{Ar}-\mathrm{H})$; EI-MS (70 eV) m/z (\%): $402\left(\mathrm{M}^{+}, 5\right), 366$ (67), 240 (16), 175 (8), 161 (5), 145 (8) 126 (100), 111 (9); IR (KBr) v: $3057(\mathrm{Ar}-\mathrm{H}), 2926,1592,1480,1461,1294,1164,1102$ $(\mathrm{C}-\mathrm{O}-\mathrm{C}), 1079,789 \mathrm{~cm}^{-1}$. Anal. calcd for $\mathrm{C}_{15} \mathrm{H}_{10^{-}}$ $\mathrm{Cl}_{3} \mathrm{~N}_{3} \mathrm{O}_{2} \mathrm{~S}$ : C 44.74, H 2.50, N 10.44; found $\mathrm{C} 45.01, \mathrm{H}$ 2.30, N 10.57 .

2-(6-氯吡啶-3-甲硫基)-5-(2-氯苯氧亚甲基)-1,3,4啞二唑(VI): 白色固体, 产率 65\%. m.p. 54.1 56.1 ${ }^{\circ} \mathrm{C}$; ${ }^{1} \mathrm{H}$ NMR (400 MHz, $\left.\mathrm{CDCl}_{3}\right) \delta: 4.43\left(\mathrm{~s}, 2 \mathrm{H}, \mathrm{SCH}_{2}\right), 5.28$ (s, $\left.2 \mathrm{H}, \mathrm{OCH}_{2}\right), 7.00 \sim 8.47(\mathrm{~m}, 7 \mathrm{H}, \mathrm{Ar}-\mathrm{H})$; IR $(\mathrm{KBr}) v: 3062$ $(\mathrm{Ar}-\mathrm{H}), 2938,1584,1480,1382,1161,1100(\mathrm{C}-\mathrm{O}-\mathrm{C})$, 1061, 1008, $748 \mathrm{~cm}^{-1}$. Anal. calcd for $\mathrm{C}_{15} \mathrm{H}_{11} \mathrm{Cl}_{2} \mathrm{~N}_{3} \mathrm{O}_{2} \mathrm{~S}: \mathrm{C}$ 48.93, H 3.01, N 11.41; found C 49.21, H 2.85, N 11.36.

2-(6-氯吡啶-3-甲硫基)-5-(4-氯苯氧亚甲基)-1,3,4噁二唑(VII): 白色固体, 产率 73\%. m.p. 97.0 98.7 ${ }^{\circ} \mathrm{C}$; ${ }^{1} \mathrm{H}$ NMR (400 MHz, $\mathrm{CDCl}_{3}$ ) $\delta: 4.43$ (s, 2H, $\left.\mathrm{SCH}_{2}\right), 5.19$ (s, $\left.2 \mathrm{H}, \mathrm{OCH}_{2}\right), 6.92 \sim 8.47(\mathrm{~m}, 7 \mathrm{H}, \mathrm{Ar}-\mathrm{H})$; IR $(\mathrm{KBr}) v: 3059$ $(\mathrm{Ar}-\mathrm{H}), 2993,2936,1586,1491,1459,1288,1161,1103$ $(\mathrm{C}-\mathrm{O}-\mathrm{C}), 821 \mathrm{~cm}^{-1}$. Anal. calcd for $\mathrm{C}_{15} \mathrm{H}_{11} \mathrm{Cl}_{2} \mathrm{~N}_{3} \mathrm{O}_{2} \mathrm{~S}: \mathrm{C}$ 48.93, H 3.01, N 11.41; found C 49.23, H 2.92, N 11.51 .

2-(6-氯吡啶-3-甲硫基)-5-(3-氯苯氧亚甲基)-1,3,4啞二唑(VIII): 白色固体, 产率 65\%. m.p. 61.5 63.2 ${ }^{\circ} \mathrm{C}$; ${ }^{1} \mathrm{H}$ NMR (400 MHz, $\left.\mathrm{CDCl}_{3}\right) \delta: 4.44$ (s, 2H, $\mathrm{SCH}_{2}$ ), $5.20\left(\mathrm{~s}, 2 \mathrm{H}, \mathrm{OCH}_{2}\right), 6.88 \sim 8.47(\mathrm{~m}, 7 \mathrm{H}, \mathrm{Ar}-\mathrm{H})$; IR (KBr) v: $3059(\mathrm{Ar}-\mathrm{H}), 2995,2934,1598,1485,1463,1382$, 1172, $1104(\mathrm{C}-\mathrm{O}-\mathrm{C}), 1021,901 \mathrm{~cm}^{-1}$. Anal. calcd for $\mathrm{C}_{15} \mathrm{H}_{11} \mathrm{Cl}_{2} \mathrm{~N}_{3} \mathrm{O}_{2} \mathrm{~S}$ : C 48.93, H 3.01, N 11.41; found C 49.27, H 3.11, N 11.28.

2-(2-氯噻唑-5-甲硫基)-5-(2,4-二氯苯氧亚甲 基)-1,3,4-㤠二唑(IX)：白色固体，产率 75\%. m.p. 67.0 $68.5{ }^{\circ} \mathrm{C} ;{ }^{1} \mathrm{H}$ NMR (400 MHz, $\left.\mathrm{CDCl}_{3}\right) \delta: 4.62$ (s, 2H, $\left.\mathrm{SCH}_{2}\right), 5.28\left(\mathrm{~s}, 2 \mathrm{H}, \mathrm{OCH}_{2}\right), 7.02 \sim 7.55$ (m, 4H, Ar-H); IR (KBr) v: 3099, 2976, 2929, 1581, 1469, 1158, 1049 (C$\mathrm{O}-\mathrm{C}) \mathrm{cm}^{-1}$. Anal. calcd for $\mathrm{C}_{13} \mathrm{H}_{8} \mathrm{Cl}_{3} \mathrm{~N}_{3} \mathrm{O}_{2} \mathrm{~S}_{2}$ : C 38.20, $\mathrm{H}$ 1.97; N 10.28; found C 38.31, H 1.91, N 10.49.

2-(2-氯噻唑-5-甲硫基)-5-(2-氯苯氧亚甲基)-1,3,4噁二唑 $(\mathbf{X})$ : 白色固体, 产率 73\%. m.p. 63.9 66.0 ${ }^{\circ} \mathrm{C}$; ${ }^{1} \mathrm{H}$ NMR (400 MHz, $\left.\mathrm{CDCl}_{3}\right) \delta: 4.60\left(\mathrm{~s}, 2 \mathrm{H}, \mathrm{SCH}_{2}\right), 5.30(\mathrm{~s}$, $\left.2 \mathrm{H}, \mathrm{OCH}_{2}\right), 6.98 \sim 7.54(\mathrm{~m}, 5 \mathrm{H}, \mathrm{Ar}-\mathrm{H})$; EI-MS (70 eV) $\mathrm{m} / \mathrm{z}$ (\%): $373\left(\mathrm{M}^{+}, 9\right), 338$ (35), 246 (10), 141 (11), 132 (100),
127.5 (17), 111 (14), 77 (6); IR (KBr) v: 3097, 3007, 2954 $(\mathrm{C}-\mathrm{H}), 1581,1480,1172,1048(\mathrm{C}-\mathrm{O}-\mathrm{C}) \mathrm{cm}^{-1}$. Anal. calcd for $\mathrm{C}_{13} \mathrm{H}_{9} \mathrm{Cl}_{2} \mathrm{~N}_{3} \mathrm{O}_{2} \mathrm{~S}_{2}$ : C 41.72, $\mathrm{H}$ 2.42, $\mathrm{N}$ 11.23; found $\mathrm{C} 42.08, \mathrm{H} 2.24, \mathrm{~N} 11.37$.

2-(2-氯噻唑-5-甲硫基)-5-(4-氯苯氧亚甲基)-1,3,4啞二唑(XI): 白色固体, 产率 80\%. m.p. 91.2 94.0 ${ }^{\circ} \mathrm{C}$; ${ }^{1} \mathrm{H}$ NMR (400 MHz, $\mathrm{CDCl}_{3}$ ) $\delta: 4.61\left(\mathrm{~s}, 2 \mathrm{H}, \mathrm{SCH}_{2}\right), 5.21$ (s, $\left.2 \mathrm{H}, \mathrm{OCH}_{2}\right), 6.93 \sim 7.55(\mathrm{~m}, 5 \mathrm{H}, \mathrm{Ar}-\mathrm{H})$; IR (KBr) v: 3006, 2961, 1583, 1493, 1447, 1161, $1061(\mathrm{C}-\mathrm{O}-\mathrm{C}) \mathrm{cm}^{-1}$. Anal. calcd for $\mathrm{C}_{13} \mathrm{H}_{9} \mathrm{Cl}_{2} \mathrm{~N}_{3} \mathrm{O}_{2} \mathrm{~S}_{2}$ : C 41.72, $\mathrm{H}$ 2.42, N 11.23; found $\mathrm{C} 42.07, \mathrm{H} 2.37, \mathrm{~N} 11.30$.

2-(2-氯噻唑-5-甲硫基)-5-(3-氯苯氧亚甲基)-1,3,4啞二唑(XII): 白色固体，产率 74\%. m.p. 80.1 81.6 ${ }^{\circ} \mathrm{C}$; ${ }^{1} \mathrm{H}$ NMR (400 MHz, $\left.\mathrm{CDCl}_{3}\right) \delta: 4.62\left(\mathrm{~s}, 2 \mathrm{H}, \mathrm{SCH}_{2}\right), 5.22$ (s, $\left.2 \mathrm{H}, \mathrm{OCH}_{2}\right), 6.89 \sim 7.55(\mathrm{~m}, 5 \mathrm{H}, \mathrm{Ar}-\mathrm{H})$; IR (KBr) v: 3103, $3076(\mathrm{Ar}-\mathrm{H}), 2965,1585,1478,1456,1158,1043(\mathrm{C}-$ $\mathrm{O}-\mathrm{C}) \mathrm{cm}^{-1}$. Anal. calcd for $\mathrm{C}_{13} \mathrm{H}_{9} \mathrm{Cl}_{2} \mathrm{~N}_{3} \mathrm{O}_{2} \mathrm{~S}_{2}: \mathrm{C} 41.72, \mathrm{H}$ 2.42, N 11.23; found C 41.93, H 2.36, N 11.41.

\section{4 晶体结构测定}

为了对该系列化合物分子结构作进一步的验证，从 二氯甲烷中培养了化合物 $\mathbf{I}$ 单晶用于 $\mathrm{X}$ 射线单晶衍射实 验. 选取尺寸为 $0.30 \mathrm{~mm} \times 0.20 \mathrm{~mm} \times 0.20 \mathrm{~mm}$ 的无色晶 体, 测定温度为 $293 \mathrm{~K}$, 在 Brucker APEX CCD Dector 型 $\mathrm{X}$ 射线单晶衍射仪上，采用石墨单色化的 Mo $\mathrm{K} \alpha$ $(\lambda=0.071073 \mathrm{~nm})$ 射线, 以 $\omega / 2 \theta$ 扫描方式在 $1.48^{\circ}<\theta<$ $26.00^{\circ}$ 范围内共收集到 4655 个衍射点, 其中独立衍射点 数为 $2544\left(R_{\text {int }}=0.0467\right)$, 收集的衍射数据使用 SAINT 软件进行还原, 经验吸收校正在 SADAB 程序上进行 $\left(T_{\min }=0.9153, T_{\max }=0.8768\right)$. 晶体结构采用直接法使用 SHELXL-97 程序解出, 对 $F^{2}$ 进行全矩阵最小二乘法修 正. 最终的偏离因子为 $R=0.0419, R_{\mathrm{w}}=0.1130$, 差值电 子云密度的最高和最低峰为 268 和 $-250 \mathrm{e} \bullet \mathrm{nm}^{-3}$. 晶体 结构分析表明，化合物晶体为三斜晶系，空间群 $P-1$, 晶胞参数为 $a=0.62729(5) \mathrm{nm}, b=0.81448(6) \mathrm{nm}, c=$ 1.40994(11) nm, $\alpha=85.5200(10)^{\circ}, \beta=77.7930(10)^{\circ}, \gamma=$ $68.6370(10)^{\circ}, Z=2, V=1.13318(15) \mathrm{nm}^{3}, D_{\mathrm{c}}=1.544$ $\mathrm{Mg} / \mathrm{m}^{3}, \mu=0.450 \mathrm{~mm}^{-1}, F(000)=312$. 数据存于英国剑 桥数据中心, $\mathrm{CCDC}$ 号为 660221 .

\section{References}

[1] Gibson, M. S. Tetrahedron 1962, 18, 1377.

[2] Cao, S.; Qian, X. H.; Song, G. H.; Huang, Q. C. J. Fluorine Chem. 2002, 117, 63 .

[3] Ishida, H.; Isami, S.; Matsumura, T.; Umehara, H.; Yamashita, Y.; Kajita, J.; Fuse, E.; Kiyoi, H.; Naoe, T.; Akinaga, S.; Shiotsu, U.; Arai, H. Bioorg. Med. Chem. Lett. 2008, 18, 5472.

[4] Zhang, L.-R.; Liu, Z.-J.; Zhang, H.; Sun, J.; Luo, Y.; Zhao, T.-T.; 
Gong, H.-B. Bioorg. Med. Chem. 2012, 20, 3615.

[5] Patel, N. B.; Purohit, A. C.; Rajani, D. P.; Moo-Puc, R.; Rivera, G. Eur. J. Med. Chem. 2013, 62, 677.

[6] Liu, Z. M.; Yang, G. F.; Qian, X. H. J. Chem. Technol. Biotechnol. 2001, 76, 1154.

[7] Wang, W. Y.; Zhao, W. G.; Li, Z. M. Chem. Res. Chin. Univ. 2004, 20,543 .

[8] Basarab, G. S.; Manchester, J. I.; Bist, S.; Boriack-Sjodin, Dangel, B.; Illingworth, R.; Sherer, B. A.; Sriram, S.; Uria-Nickelsen, M.; Eakin, A. E. J. Med. Chem. 2013, 56, 8712.

[9] Wu, X.; Zhu, C.; Lv, Z.; Wei, C.; Liao, X. Chin. J. Org. Chem. 2011, 31, 824 (in Chinese).

(武现丽, 朱春风, 吕志丹, 魏成事, 廖新成, 有机化学, 2011, 31, 824.)

[10] Khan, M. S.; Gita, C.; Asad, M. M. Indian J. Chem. 2004, 43B,
1302.

[11] Zheng, X. M.; Li, Z.; Wang, Y. L.; Chen, W. D.; Huang, Q. C.; Liu, C. X.; Song, G. H. J. Fluorine Chem. 2003, 123, 163.

[12] Ji, Z.; Liu, F.; Zhang, Z.; Li, F.; Jiang, L. Chin. J. Org. Chem. 2012, 32, 2129 (in Chinese).

(纪增臣, 刘峰, 张泽远, 李付博, 姜林, 有机化学, 2012, 32, 2129.)

[13] Chen, T.; Shen, P.; Li, Y. J.; He, H. W. J. Fluorine Chem. 2006, 127, 291.

[14] Cui, Z.; Li, Y.; Ling, Y.; Huang, J.; Cui, J.; Wang, R.; Yang, X. Eur.J. Med.Chem. 2010, 45, 5576.

[15] Chen, H. S.; Li, Z. M.; Han, Y. F. J. Agric. Food Chem. 2000, 48, 5312 .

[16] Burbuliene, M. M.; Jakubkiene, V.; Mekuskiene, G.; Udrenaite, E.; Smicius, R.; Vainilavicius, P. Farmaco 2004, 59, 767.

(Zhao, C.) 